\title{
Comparative analysis of the athletes' functional condition in cyclic and situational sports
}

\author{
Podrigalo O.O. ${ }^{1,2 \mathrm{ABCD}}$, Borisova O.V. ${ }^{1 \mathrm{ABCD}}$, Podrigalo L.V. ${ }^{2 \mathrm{ABCD}}$, Iermakov S.S. ${ }^{3 \mathrm{ABCD}}$, Romanenko V.V. ${ }^{2 \mathrm{ABCDE}}$, \\ Podavalenko O.V. ${ }^{2 \mathrm{ABCDE}}$, Volodchenko O.A. ${ }^{2 \mathrm{ABCDE}}$, Volodchenko J.O. ${ }^{2 \mathrm{ABCDE}}$ \\ ${ }^{1}$ National University of Ukraine on Physical Education and Sport, Kiev, Ukraine \\ ${ }^{2}$ Kharkov State Academy of Physical Culture, Kharkov, Ukraine \\ ${ }^{3}$ Gdansk University of Physical Education and Sport, Gdansk, Poland
}

Authors' Contribution: A - Study design; B - Data collection; C - Statistical analysis; D - Manuscript Preparation; E - Funds Collection.

\begin{abstract}
Purpose:

The athletic potential of athletes depends on functional condition parameters. They determine physical and mental performance. Their evaluation is part of the athletes' condition monitoring in many sports. The purpose of the study was the comparative analysis of the athletes' functional condition in cyclic and situational sports.

Material: $\quad$ The study involved 31 students from specialized sport school aged 16-17. The participants were divided into two groups depending on the sport: $1^{\text {st }}$ group - 20 martial arts athletes (Greco-Roman wrestling, freestyle wrestling, judo), $2^{\text {nd }}$ group -11 water sports athletes (swimming). The level of sportsmanship was Candidates and Masters of Sports. The following tests were applied: determination of the individual minute duration (IM) $(\mathrm{s})$, measuring of a $10 \mathrm{~cm}$ segment, $(\mathrm{cm})$, and determination of simple hand-eye coordination (SHEyC), (s), and simple hand-ear coordination (SHEaC), (s), "Figures memorizing" technique, solution of 10 sums.

Results: $\quad$ The IM test results reflect the predominance of earlier completion of the test. This tendency more expressed in martial arts athletes. The results of measuring a segment were lower in all tests in the $1^{\text {st }}$ group and in 9 tests in the $2^{\text {nd }}$ group. The vision reaction in 4 tests out of 10 was better in martial arts athletes. The response to the auditory stimulus in 5 tests out of 10 and the average result was better in martial arts athletes. The results of figures memorizing test were most often average. The swimmers memorize significantly fewer figures according to 3 tests. The results of the solution of sums were similar.

Conclusions: The use of a battery test allows giving a comparative analysis of the functional condition of different sports athletes. The stability of IM test results indicates sufficient adaptation reserves of the participants. The analysis of the results of the measuring of segment test allows evaluating the ability to spatial orientation as an important for success quality in martial arts and water sports. The importance of the response rate for the success of both martial arts athletes and swimmers confirmed. The martial arts athletes' results were significantly better. This confirms their ability to concentrate, to stay focused longer. The results allow considering the response rate to an auditory stimulus as an important indicator for predicting success. The figures memorizing test also reflects the specificity of sports. It illustrates the ability of athletes to control the environment, manage the situation. This ability is significantly better in martial arts athletes. The dynamics of solving sums results suggest some complexity with a long focusing on the swimmers and better parameters in martial arts athletes. The simplicity, accessibility and informative nature of the used tests suggest their use in the athletes' functional condition monitoring.

Keywords: martial arts, swimming, athletes, psychophysiological tests, functional condition.
\end{abstract}

\section{Introduction}

Functional condition considered one of the important criteria that determine a person's physical and mental performance. The sporting potential is largely dependent on these parameters. They have a significant effect on the possible increase in athletes' skills. The evaluation and analysis of functional condition by means of special tests is part of athletes' condition monitoring in different sports.

Ziagkas et al [1] determine that response time is one of the person's abilities that determines sports activities and is an important factor for the victory. Special training improves this parameter. Ferreira et al [2] obtained the similar data. The authors consider that response time is an important factor in martial arts. It suggested to use response time as a preparation criterion and athletes' monitoring tool [3].

(c) Podrigalo O.O., Borisova O.V., Podrigalo L.V., lermakov S.S., Romanenko V.V., Podavalenko O.V., Volodchenko O.A.,

Volodchenko J.O., 2019

doi:10.15561/20755279.2019.0606
Krawczyk et al [4] evaluated the psychophysiological condition of football goalkeepers. It was confirmed the high parameters of response time, choice response time, the ability of athletes to predict the development of the game situation. Romanenko et al [5] obtained the similar data. The application of choice response as a screening test in martial arts athletes allows recommending this test for monitoring.

Table tennis is a sport in which players perform technical actions at a high rate [6]. The response rate and processing time are key factors that influence on player's success. The results of Sanabria et al [7] demonstrate the different response rates in different sports. This parameter is better in sports with extrinsic rhythm (football) than in sports with independent rhythm (triathlon).

Monfort et al [8] used visual and verbal memory parameters, response rate, and information processing time as criteria for evaluating the football players' progress. The evaluation of visual-spatial memory 
provides information for neuromuscular control disorders.

Cognitive abilities determine the progress of athletic achievement [9]. Among these are attention, memory, information processing rate, spatial orientation. Training these skills increases the mental performance of the athlete, helps to make more decisions that are effective in the game.

The response rate in basketball has particular importance, especially for predicting and making decisions. Leonte et al [10] compared the results of response time in female athletes with results of nonathletes. It confirmed that there are significant differences between the groups.

Albuquerque et al [11] evaluated the correlation of functional parameters of football players. Players with better tactical characteristics responded more quickly to the given tasks. Biscaia et al [12] analyzed differences between handball players with different levels of training in speed and accuracy of information processing. More experienced players have better response time and are more accurate. Increasing athletic experience leads to reduced information-processing time, especially in difficult situations.

Yuksel et al [13] investigated the response time of national badminton teams' athletes to simple visual and auditory stimuli. It is emphasized the importance of this factor for success along with technique and tactical data.

Athletes in sports related to the ball or racket are under constant time pressure [14]. Optimal visualmotor characteristics of athletes mostly related to visual perception and, to a lesser extent, to motor processes.

Thus, the available data make allow considering the direction of work devoted to the study of psychophysiological features of different sports athletes.

The purpose of the study is a comparative analysis of the athletes' functional condition in cyclic and situational sports.

\section{Materials and methods}

\section{Participants}

The study involved 31 students from a specialized sport school aged 16-17 years. The participants were divided into two groups, depending on the sport: 1st group - 20 martial arts athletes (Greco-Roman wrestling, freestyle wrestling, judo), 2nd group - 11 water sports athletes (swimming). The level of sportsmanship was Candidates and Masters of Sports.

The study design

The following battery tests were performed: determination of the individual minute duration (IM) (s), measuring of a $10 \mathrm{~cm}$ segment, $(\mathrm{cm})$, and determination of simple hand-eye coordination (SHEyC), (s), and simple hand-ear coordination ( $\mathrm{SHEaC})$, (s), "Figures memorizing" technique (abs), solution of 10 sums (\% of correct answers).

IM duration. The participant counted seconds silently (from 1 to 60); started on a signal of the researcher and said aloud the number 60 . The stopwatch also fixed the time. The test repeated 10 times. The IM duration fixed.
Measuring of the segment. The $10 \mathrm{~cm}$ segment drawn on a piece of paper. Then the same segment drawn without visual control. Its length fixed. The test repeated 10 times. The length of the segment fixed.

Determination of simple hand-eye coordination (SHEyC) and simple hand-ear coordination (SHEaC) performed using the chronoreflexometry. The study repeated ten times. The response rate fixed.

"Figures memorizing" technique. The participants shown tables with 10 double figures for 30 seconds to remember. The participants recorded these figures after the table put off. The test repeated 10 times. The number of correctly remembered figures fixed.

The solution of 10 sums. Participants asked to solve mentally 10 sums with double figures. The test repeated 4 times. The percentage of correctly solved sums fixed.

\section{Statistical analysis}

Statistical analysis performed using licensed MS Excel. The following indicators of descriptive statistics were determined: arithmetic means, standard deviation, and error of the mean. The significance of differences in the groups evaluated using the Student's t-test $(t)$ and the non-parametric Rosenbaum's test (Q).

\section{Results}

The results are given in Tables 1-3.

The individual minute was shorter than the actual one in athletes of the $1^{\text {st }}$ group. This was determined in 8 tests out of 10. In $2^{\text {nd }}$ and $3^{\text {rd }}$ tests the individual minute was more than the actual one. The count lasted longer than necessary. This tendency less expressed in swimmers. They finished the count earlier in 6 tests out of 10 . The number of later test completions was 3. Swimmers accurately completed the test only in 1 test.

The comparison of the groups confirmed some differences in the performance of this test. The results of the $2^{\text {nd }}$ group were significantly higher in performing the 5 th test $(\mathrm{t}=2.14, \mathrm{Q}=9, \mathrm{p}<0.05)$, as well as the average test result $(\mathrm{Q}=8, \mathrm{p}<0.05)$.

The results of segment measuring were less than the standard in all tests in the $1^{\text {st }}$ group and 9 tests in the 2 nd group. The results of the $8^{\text {th }}$ test in the 2 nd group were more than the standard segment. There were discovered differences between groups. The results of martial art athletes were significantly higher $(\mathrm{t}=2.42, \mathrm{p}<0.05)$ in the $1^{\text {st }}$ test. The results of water sports athletes were higher in $7^{\text {th }}$ and 8 th tests, respectively, $\mathrm{Q}=8,(\mathrm{p}<0.05)$ and $\mathrm{t}=$ $3.90, \mathrm{Q}=13,(\mathrm{p}<0.05)$.

The response to the visual stimulus in 6 tests out of 10 had no significant differences between the groups. The results of martial art athletes were significantly better in $2^{\text {nd }}, 7^{\text {th }}, 8^{\text {th }}$ and $10^{\text {th }}$ tests, respectively $(\mathrm{t}=2.34, \mathrm{Q}=7, \mathrm{p}$ $<0.05),(\mathrm{t}=3.06, \mathrm{Q}=9, \mathrm{p}<0.05) .(\mathrm{t}=2.93, \mathrm{p}<0.05),(\mathrm{t}=$ $2.40, \mathrm{p}<0.05)$.

The response rate to the auditory stimulus had more expressed differences. Significantly better response rate determined according to the following tests: in $2^{\text {nd }}$ test $(\mathrm{t}=2.84, \mathrm{Q}=9, \mathrm{p}<0.05)$, in $3^{\text {rd }}$ test $(\mathrm{t}=2.99, \mathrm{Q}=18, \mathrm{p}$ $<0.05)$, in $4^{\text {th }}$ test $\left.(\mathrm{t}=2.31, \mathrm{Q})=9, \mathrm{p}<0.05\right)$, in $5^{\text {th }}$ test $(\mathrm{t}=$ 
Table 1. Results of psychophysiological testing of cyclic and situational sports athletes

\begin{tabular}{|c|c|c|}
\hline Parameters & $1^{\text {st }}$ group $(n=20)$ & $2^{\text {nd }}$ group $(n=11)$ \\
\hline IM duration, 1 test, s & $56.42 \pm 4.03$ & $59.04 \pm 3.45$ \\
\hline IM duration, 2 test, s & $60.76 \pm 1.61$ & $65.34 \pm 2.29$ \\
\hline IM duration, 3 test, s & $60.78 \pm 1.73$ & $63.90 \pm 1.61$ \\
\hline IM duration, 4 test, s & $58.86 \pm 1.44$ & $60.32 \pm 2.01$ \\
\hline IM duration, 5 test, s & $57.11 \pm 1.62$ & $61.78 \pm 1.45^{1}$ \\
\hline IM duration, 6 test, s & $57.96 \pm 1.1$ & $59.66 \pm 1.24$ \\
\hline IM duration, 7 test, s & $57.34 \pm 1.10$ & $57.36 \pm 1.60$ \\
\hline IM duration, 8 test, s & $58.54 \pm 1.43$ & $59.01 \pm 0.89$ \\
\hline IM duration, 9 test, s & $59.29 \pm 1.12$ & $58.25 \pm 1.23$ \\
\hline IM duration, 10 test, s & $58.83 \pm 1.13$ & $59.68 \pm 0.93$ \\
\hline IM duration, average value, s & $58.61 \pm 0.56$ & $60.43 \pm 0.93$ \\
\hline Measuring of segment, 1 test, $\mathrm{cm}$ & $8.09 \pm 0.45$ & $6.23 \pm 0.62^{1}$ \\
\hline Measuring of segment, 2 test, $\mathrm{cm}$ & $8.38 \pm 0.40$ & $7.58 \pm 0.51$ \\
\hline Measuring of segment, 3 test, $\mathrm{cm}$ & $9.16 \pm 0.33$ & $8.73 \pm 0.44$ \\
\hline Measuring of segment, 4 test, $\mathrm{cm}$ & $9.24 \pm 0.32$ & $9.06 \pm 0.42$ \\
\hline Measuring of segment, 5 test, $\mathrm{cm}$ & $9.25 \pm 0.25$ & $9.67 \pm 0.32$ \\
\hline Measuring of segment, 6 test, $\mathrm{cm}$ & $9.45 \pm 0.32$ & $9.63 \pm 0.24$ \\
\hline Measuring of segment, 7 test, $\mathrm{cm}$ & $9.51 \pm 0.20$ & $9.83 \pm 0.30$ \\
\hline Measuring of segment, 8 test, $\mathrm{cm}$ & $9.42 \pm 0.15$ & $10.17 \pm 0.12^{1}$ \\
\hline Measuring of segment, 9 test, $\mathrm{cm}$ & $9.46 \pm 0.15$ & $9.44 \pm 0.33$ \\
\hline Me 2asuring of segment, 10 test, $\mathrm{cm}$ & $9.76 \pm 0.10$ & $9.97 \pm 0.15$ \\
\hline Measuring of segment, average value, $\mathrm{cm}$ & $9.17 \pm 0.21$ & $9.03 \pm 0.22$ \\
\hline
\end{tabular}

Notes. 1 - differences with the 1 st group are significant $(p<0.05)$

Table 2. Results of response rate evaluation of cyclic and situational sports athletes

\begin{tabular}{lll}
\hline Parameters & $\mathbf{1}^{\text {st }}$ group $(\mathbf{n = 2 0})$ & $\mathbf{2}^{\text {nd }} \mathbf{g r o u p}$ (n=11) \\
\hline SHEyC, 1 test, s & $0.24 \pm 0.01$ & $0.24 \pm 0.01$ \\
SHEyC, 2 test, s & $0.21 \pm 0.01$ & $0.26 \pm 0.02^{1}$ \\
SHEyC, 3 test, s & $0.20 \pm 0.01$ & $0.18 \pm 0.02$ \\
SHEyC, 4 test, s & $0.21 \pm 0.01$ & $0.22 \pm 0.01$ \\
SHEyC, 5 test, s & $0.22 \pm 0.01$ & $0.24 \pm 0.01$ \\
SHEyC, 6 test, s & $0.21 \pm 0.01$ & $0.22 \pm 0.01$ \\
SHEyC, 7 test, s & $0.19 \pm 0.01$ & $0.24 \pm 0.01^{1}$ \\
SHEyC, 8 test, s & $0.19 \pm 0.01$ & $0.24 \pm 0.01^{1}$ \\
SHEyC, 9 test, s & $0.21 \pm 0.01$ & $0.24 \pm 0.01$ \\
SHEyC, 10 test, s & $0.20 \pm 0.01$ & $0.24 \pm 0.01^{1}$ \\
SHEyC, average value, s & $0.21 \pm 0.01$ & $0.23 \pm 0.01$ \\
SHEaC, 1 test, s & $0.19 \pm 0.01$ & $0.21 \pm 0.02$ \\
SHEaC, 2 test, s & $0.17 \pm 0.01$ & $0.22 \pm 0.02^{1}$ \\
SHEaC, 3 test, s & $0.17 \pm 0.01$ & $0.21 \pm 0.01^{1}$ \\
SHEaC, 4 test, s & $0.16 \pm 0.01$ & $0.21 \pm 0.02^{1}$ \\
SHEaC, 5 test, s & $0.17 \pm 0.01$ & $0.20 \pm 0.01^{1}$ \\
SHEaC, 6 test, s & $0.19 \pm 0.01$ & $0.21 \pm 0.01$ \\
SHEaC, 7 test, s & $0.18 \pm 0.01$ & $0.21 \pm 0.01^{1}$ \\
SHEaC, 8 test, s & $0.17 \pm 0.01$ & $0.21 \pm 0.02$ \\
SHEaC, 9 test, s & $0.15 \pm 0.01$ & $0.19 \pm 0.02$ \\
SHEaC, 10 test, s & $0.16 \pm 0.01$ & $0.18 \pm 0.02$ \\
SHEaC, average value, s & $0.17 \pm 0.01$ & $0.20 \pm 0.01^{1}$ \\
\hline
\end{tabular}

Notes. 1 - differences with the $1^{\text {st }}$ group are significant $(p<0.05)$ 
Table 3. Results of memory evaluation of and analytical abilities of cyclic and situational sports athletes

\begin{tabular}{|c|c|c|}
\hline Parameters & $1^{\text {st }}$ group $(n=20)$ & $2^{\text {nd }}$ group $(n=11)$ \\
\hline Figures memorizing, 1 test, abs & $5.41 \pm 0.51$ & $5.75 \pm 0.80$ \\
\hline Figures memorizing, 2 test, abs & $7.53 \pm 0.39$ & $5.63 \pm 0.18^{1}$ \\
\hline Figures memorizing, 3 test, abs & $6.65 \pm 0.40$ & $5.13 \pm 0.40^{1}$ \\
\hline Figures memorizing, 4 test, abs & $5.59 \pm 0.51$ & $6.13 \pm 1.03$ \\
\hline Figures memorizing, 5 test, abs & $6.76 \pm 0.32$ & $5.88 \pm 0.48$ \\
\hline Figures memorizing, 6 test, abs & $6.82 \pm 0.45$ & $6.00 \pm 0.78$ \\
\hline Figures memorizing, 7 test, abs & $6.12 \pm 0.55$ & $6.38 \pm 0.68$ \\
\hline Figures memorizing, 8 test, abs & $6.29 \pm 0.46$ & $4.88 \pm 0.61$ \\
\hline Figures memorizing, 9 test, abs & $6.59 \pm 0.33$ & $4.75 \pm 0.56^{1}$ \\
\hline Figures memorizing, 10 test, abs & $7.18 \pm 0.39$ & $6.63 \pm 0.53$ \\
\hline Figures memorizing, average value, abs & $6.49 \pm 0.21$ & $5.71 \pm 0.42$ \\
\hline Solving sums, 1 test, $\%$ & $69.44 \pm 10.86$ & $76.67 \pm 14.10$ \\
\hline Solving sums, 2 test, $\%$ & $38.89 \pm 11.49$ & $45.56 \pm 16.60$ \\
\hline Solving sums, 3 test, $\%$ & $92.50 \pm 6.21$ & $93.33 \pm 8.31$ \\
\hline Solving sums, 4 test, $\%$ & $91.67 \pm 6.51$ & $77.78 \pm 13.86$ \\
\hline Solving sums, average value, $\%$ & $73.13 \pm 10.45$ & $73.33 \pm 14.74$ \\
\hline
\end{tabular}

Notes. 1 - differences with group 1 are significant $(p<0.05)$

$2.20, \mathrm{p}<0.05)$, in $7^{\text {th }}$ tests $(\mathrm{t}=2.03, \mathrm{Q}=10, \mathrm{p}<0.05)$, in $8^{\text {th }}$ test $(\mathrm{Q}=8, \mathrm{p}<0.05)$ and according to the average result for all tests $(t=2.40, Q=11, p<0.05)$.

The results of the figures memorizing test in all tests were below the maximum possible result. The martial art athletes had an average result in 8 tests (5-6 figures) and result above the average in 2 tests (7-9 figures). The swimmers had average results in 8 tests and low results in 2 tests (3-4 figures). The water sport athletes remembered significantly fewer figures than martial art athletes in $2^{\text {nd }}$ test $(\mathrm{t}=4.38, \mathrm{p}<0.05)$, in $3^{\text {rd }}$ test $(\mathrm{t}=2.69, \mathrm{p}<0.05)$, and in $9^{\text {th }}$ test $(\mathrm{t}=2.83, \mathrm{p}<0,05)$.

The results of the solving sums test in the groups were similar. Participants in $1^{\text {st }}$ test demonstrated the average result, in $2^{\text {nd }}$ test demonstrated a low result. The results of $3^{\text {rd }}$ and $4^{\text {th }}$ tests were quite high, especially in the $1^{\text {st }}$ group. The substantially better solving sums were fixed in participants of the $2^{\text {nd }}$ group in $1^{\text {st }}$ test $(Q=9, p<0.05)$ and in 2 test $(\mathrm{Q}=14, \mathrm{p}<0.05)$.

\section{Discussion}

The available sources reveal that most commonly used in sports practice is the study of response rate. This stipulated by the informative nature of this criterion for evaluation of the athletes' functional condition, their success prediction

We applied a battery of six tests. The ability for temporal and spatial orientation, short-term memory, logic, and analytical capabilities evaluated in addition to the response rate. The applied tests provide a comprehensive analysis of the psychophysiological features of athletes.

The comparison of different sports athletes often used in sports science. A similar design used in the work of Krenn et al [15]. The authors compared the athletes' executive functions of static, interceptive and strategic sports. The strategic sports athletes in comparison with static sports had better indicators of mean response time, cognitive shifts, and memory.

Aksoy et al [16] used a similar design. The authors studied the psychophysiological and physiological parameters of playing sports and martial arts athletes. The expressed differences in athletes' response rate, power, and anaerobic abilities confirmed.

The repeat of the tests is also quite common in sports research. Luis del Campo et al [17] used a succession repeat of ten tests to evaluate the motor performance of boxers. The importance of response rate and motor skills of athletes for success is determined.

Hromcik et al [18] evaluated the dynamics of the accuracy of sensorimotor skills, which play an important role in ball games. Participants passed a computer test for 45 minutes. The response rate and the movement time checked with the different numbers of tasks consisting of hitting the target. The importance of motion synchronization for the prediction emphasized. Analysis of the results of almost all tests allows us to consider the condition of the athletes similar. Our data are close Le Mansec et al [19]. The authors evaluated the dynamics of the response time depending on different loads. It shown that neither mental nor physical load has a significant effect on the response rate to simple stimuli.

The stability of IM test results allows speaking about the balance and strength of the nervous processes, to evaluate the adaptation reserves of the participants as sufficient and confirms the high level of athletes' health. It was determined a tendency for all participants to finish the count earlier. In comparing groups, we can conclude that this tendency more expressed in martial arts athletes. It could considered as a reflection of the influence of the specificity of the sport on the reactivity of the nervous 
system and mobilization abilities. The average result of this test in swimmers is closer to the standard than in martial arts athletes.

Our data are close to the results of Koryagina [20]. It confirmed that this test most accurately performed by playing sports athletes (volleyball players, football players) and weight lift athletes. The earlier completion of the test demonstrated by cyclic sports athletes (skiers, track and field athletes, skaters) and difficult coordination (gymnasts) sports. Basketball players, hockey players, and boxers characterized by a later completion of the test.

The measuring of the segment test evaluates the ability of athletes to spatial orientation. There are no significant differences in this quality between the athletes of the studied groups. The error of most results is 10$15 \%$, which coincides with Koryagina's data [20]. She showed that most athletes performed this test exactly (an error within 15\%). The increase in error found in skiers, wrestlers, and non-athletes. The analysis of this test results makes it possible to evaluate the ability to spatial orientation as an important quality for success in martial arts and water sports.

Response rate considered one of the most important predictors of success in many sports. De la Fuente et al [21] proposed using it as a selection criterion and predictor of success in taekwondo.

At the same time, Martinez de Quel et al [22] emphasized that there was no clear consensus that response time was a good predictor of success in martial arts. However, comparison with the results of other sports athletes confirmed that experienced martial arts athletes predict better the actions of the opponent based on information received before and during the attack.

Studies of Podrigalo et al [23] confirmed the importance of response to different stimuli as predictors of martial arts athletes' success. The specificity of the sport determines the different information content of the tests, gives the necessary information to predict the athletes' success. This information can used for athletes' condition monitoring.

The obtained results confirm the importance of the response rate for the success of all participants. The indirect confirmation of this fact is the absence of significant differences in 6 tests out of 10 for the visual stimulus. The results of martial arts athletes in the final tests were significantly better. This confirms their ability to concentrate, to keep their attention longer than swimmers.

This assumption confirmed by the analysis of the response rate to the auditory stimulus. The martial arts athletes have demonstrated the best results in 6 tests of a series and in the average result. This reflects the specificity of the sport. The starting response is the most important for the swimmer as shown by the results of the $1^{\text {st }}$ test. For martial arts athletes is important to keep constant concentration. The athletes of the $1^{\text {st }}$ group keep a high response rate in all tests of the series. The results allow us to consider the response rate to an auditory stimulus as an important indicator for predicting success.

The results obtained are close to those of Koryagina
[20]. The author demonstrated the best response rate to light in boxers, then in the order of its increase followed by weight lifter athletes, football players, and track and field athletes. The worst response to light observed in volleyball players and weight lifter athletes. The study of simple hand-ear coordination duration showed the best values of this indicator in football players, weight lifter athletes, skaters, and boxers. Then, in the order of its increase followed by hockey players, track and field athletes, volleyball players, basketball players, wrestlers, non-athletes, skiers, weight lifter athletes, and gymnasts.

In our opinion, the figures memorizing test also reflects the specificity of sports. Swimmers do not need to focus on any objects in the swimming process. The minimum number of activities performed by swimmers causes a low level of concentration on the environment. In martial arts, on the contrary, this is a significant criterion. It reflects the ability of athletes to control the environment, manage the situation. The martial arts athletes are under constant time pressure and must make decisions, take actions, and correct them according to the current situation.

The importance of such functions reported by Montuori et al [24]. Constant changes in conditions require correction of the athletes' behavior. This achieved by continuous changes between different tasks. The results showed a minimal degree of cognitive flexibility for highly specialized athletes and a maximum level for mixed performers. The authors propose to use tests to evaluate these functions in selecting process and defining a sports position.

Solving the sums test allows evaluating the logic and analytical abilities of participants. In our opinion, the results demonstrate the complexity of the task for all participants. In martial arts athletes after the average result in 1 test, there is a diminishing of results in the $2^{\text {nd }}$ test. The other tests participants solved with a good result. The zigzag results were observed in swimmers as $2^{\text {nd }}$ and $4^{\text {th }}$ tests showed diminishing of results compared to $1^{\text {st }}$ and $3^{\text {rd }}$ tests. The difference between the results suggests some difficulties with analytical abilities, extrapolation and more rigidly fixed behavioral stereotypes in swimmers and better performance in martial arts athletes. Perhaps the obtained results reflect the gradual formation of fatigue due to the full battery tests.

The results of Fard et al [25] can confirm this assumption. [25] The authors evaluated mental fatigue during long-term simple testing in athletes compared with non-athletes. It shown that athletes demonstrated greater resistance to mental fatigue than non-athletes. Therefore, it suggested that physical exercise could reduce the negative impact of mental fatigue on a long-term cognitive task.

\section{Conclusion}

The use of a battery test (response rate, spatial and temporal orientation, logic and analytical capabilities) allows giving a comparative analysis of the functional condition of different sports athletes.

The stability of IM test results indicates sufficient adaptation reserves of the participants. The analysis of the 
results of the measuring of segment test allows evaluating the ability to spatial orientation as an important for success quality in martial arts and water sports.

The importance of the response rate for the success of both martial arts athletes and swimmers was confirmed. The martial arts athletes' results were significantly better. This confirms their ability to concentrate, to stay focused longer. The results allow considering the response rate to an auditory stimulus as an important indicator for predicting success.

The figures memorizing test also reflects the specificity of sports. It illustrates the ability of athletes to control the environment, manage the situation. This ability is significantly better in martial arts athletes.

The dynamics of solving sums results suggest some complexity with a long focusing on the swimmers and better parameters in martial arts athletes.

The simplicity, accessibility and informative nature of the used tests suggest their use in the athletes' functional condition monitoring.

\section{Conflicts of Interest}

The authors declare no conflict of interest.

\section{References}

1. Ziagkas E, Zilidou V, Politopoulos N, Douka S, Tsiatsos T, Grouios G. The Effect of a 12 Week Reaction Time Training Using Active Video Game Tennis Attack on Reaction Time and Tennis Performance. In: Auer ME, Tsiatsos $\mathrm{T}$, editors. Interactive Mobile Communication Technologies and Learning, vol. 725, Cham: Springer International Publishing; 2018. P. 644-52. https://doi.org/10.1007/978-3-319-75175-7 63

2. Ferreira TV, Noce F, da Costa IT. Is there a difference by sex in simple reaction time and impulsivity in Junior Brazilian Judo Team athletes? Archives of Budo Science of Martial Arts and Extreme Sports, 2017:13:111-118.

3. ten Haaf T, van Staveren S, Iannetta D, Roelands B, Meeusen $\mathrm{R}$, Piacentini MF, et al. Changes in Choice Reaction Time During and After 8 Days Exhaustive Cycling Are Not Related to Changes in Physical Performance. International Journal of Sports Physiology and Performance 2018;13:428-33. https://doi.org/10.1123/ijspp.2017-0218

4. Krawczyk P, Bodasinski S, Bodasinska A, Slupczynski B. Level of psychomotor abilities in handball goalkeepers. Journal of Health and Physical Activity, 2018;10:64-71. https://doi.org/10.29359/BJHPA.10.3.07

5. Romanenko V, Podrigalo L, Iermakov S, Rovnaya O, Tolstoplet E, Tropin Y, et al. Functional state of martial arts athletes during implementation process of controlled activity comparative analysis. Physical ActivityReview, 2018;6:87-93. https://doi.org/10.16926/par.2018.06.12

6. Castellar C, Pradas F, Carrasco L, La Torre AD, González-Jurado JA. Analysis of reaction time and lateral displacements in national level table tennis players: are they predictive of sport performance? International Journal of Performance Analysis in Sport, 2019;19:467-77. https://doi.org/10.1080/24748668.2019.1621673

7. Sanabria D, Luque-Casado A, Perales JC, Ballester R, Ciria LF, Huertas F, et al. The relationship between vigilance capacity and physical exercise: a mixedeffects multistudy analysis. PeerJ, 2019;7:e7118. https://doi.org/10.7717/peerj.7118

8. Monfort SM, Pradarelli JJ, Grooms DR, Hutchison KA, Onate JA, Chaudhari AMW. Visual-Spatial Memory Deficits Are Related to Increased Knee Valgus Angle During a SportSpecific Sidestep Cut. Am J Sports Med, 2019;47:1488-95. https://doi.org/10.1177/0363546519834544

9. Alfonso M, Jose I. Neuroscience and Training in High Performance. Sports Revista Iberoamericana de Ciencias de la Actividad Fisica y el Deporte, 2019;8(2): 79-90. https://doi.org/10.24310/riccafd.2019.v8i2.6698

10.Leonte N, Moanta AD, Popescu O. Computerized Differences in the Simple Reaction Time Between Athlete and Non-
Athlete Female Students in UPB - comparative analysis. 15th International Scientific Conference on eLearning and Software for Education (eLSE) - New Technologies and Redesigning Learning Spaces, 2019: 3: 483-488.

11. Albuquerque MR, Gonzaga A dos Santos, Greco PJ. Association between inhibitory control and tactical performance of under-15 soccer players. Revista de Psicologia del Deporte, 2019;28(1): 63-69.

12.Biscaia P, Coelho E, Hernandez-Mendo A. Information Processing and Anticipation in Elite Handball Players: from the Beginning to the Top Level. Revista Iberoamericana de Psicologia del Ejercicio y el Deporte, 2018;13(2): 179-191.

13.Yuksel MF, Tunc GT. Examining the Reaction Times of International Level Badminton Players Under 15. Sports, 2018;6(1): e20. https://doi.org/10.3390/sports6010020

14.Huelsduenker T, Strueder HK, Mierau A. Visual Motion Processing Subserves Faster Visuomotor Reaction in Badminton Players. Medicine and Science in Sports and Exercise, 2017;49(6):1097-1110. https://doi.org/10.1249/MSS.0000000000001198

15.Krenn B, Finkenzeller T, Würth S, Amesberger G. Sport type determines differences in executive functions in elite athletes. Psychology of Sport and Exercise, 2018;38: 72-79. https://doi.org/10.1016/j.psychsport.2018.06.002

16.Aksoy Y, Agaoglu SA. The Comparison of Sprint Reaction Time and Anaerobic Power of Young Football Players, Volleyball Players and Wrestlers. Kinesiologia Slovenica, 2017;23(2): 5-14.

17.Luis del Campo V, Gonzalez Fernandez R. The Use of the Interpersonal Distance in the Motor Behavior of Boxers with Different Sport Level. Revista de Psicologia del Deporte, 2017;26(2): 87-93.

18.Hromcik A, Zvonar M, Balint G. Differences in Sensorimotor Skills between Badminton Players and Non-Athlete Adults Brain-broad. Research in Artificial Intelligence and Neuroscience, 2019;10(2): 47-54.

19.Le Mansec Y, Dorel S, Nordez A, Jubeau M. Is reaction time altered by mental or physical exertion? Eur J Appl Physiol, 2019;119:1323-35. https://doi.org/10.1007/s00421-019-04124-7

20.Koryagina UV. Perception of time and space in sports activities. M.Scientific and Publishing Center "Theory and Practice of Physical Culture and Sports"; 2006. (in Russian)

21.De la Fuente A, Gomez-Landero Rodriguez LA. Motor Differences in Cadet Taekwondo Athletes According to Competition Level. Revista Internacional de Medicina y Ciencias de la Actividad Fisica y del Deporte, 2019;19(73): 63-75. https://doi.org/10.15366/rimcafd2019.73.005 
22.Martinez de QO, Bennett SJ. Perceptual-cognitive expertise in combat sports: a narrative review and a model of perception-action. Ricyde-revista Internacional de Ciencias del Deporte, 2019;15(58): 323-338. https://doi.org/10.5232/ricyde2019.05802

23.Podrigalo L, Iermakov $\mathrm{S}$, Romanenko $\mathrm{V}$, Rovnaya O, Tropin Y, Goloha V, et al. Psychophysiological features of athletes practicing different styles of martial arts - the comparative analysis. International Journal of Applied Exercise Physiology, 2019;8:84-91. https://doi.org/10.30472/ijaep.v8i1.299
24.Montuori S, D’Aurizio G, Foti F, Liparoti M, Lardone A, Pesoli $\mathrm{M}$, et al. Executive functioning profiles in elite volleyball athletes: Preliminary results by a sport-specific task switching protocol. Human Movement Science, 2019;63:73-81. https://doi.org/10.1016/j.humov.2018.11.011

25.Fard SJ, Boroujeni ST, Lavender AP. Mental fatigue impairs simple reaction time in non-athletes more than athletes fatigue. Biomedicine Health and Behavior, 2019;7(3): 117-126. https://doi.org/10.1080/21641846.2019.1632614

\section{Information about the authors:}

Podrigalo O.O.; http://orcid.org/0000-0003-1519-5632; rovnayaolga77@ukr.net; Kharkov State Academy of Physical Culture; Klochkovskaya str. 99, Kharkov, 61022, Ukraine.

Borisova O.V.; http://orcid.org/0000-0002-2311-1921; borisova-nupesu@ukr.net; National University of Ukraine on Physical Education and Sport, Fiskulturnaya str. 1, Kiev, Ukraine

Podrigalo L.V. (Corresponding author); http://orcid.org/0000-0002-7893-524X; I.podrigalo@mail.ru; Kharkov State Academy of Physical Culture; Klochkovskaya str. 99, Kharkov, 61022, Ukraine.

lermakov S.S.; http://orcid.org/0000-0002-5039-4517; sportart@gmail.com; Gdansk University of Physical Education and Sport; Kazimierza Górskiego 1, 80-336 Gdańsk, Poland.

Romanenko V.V.; https://orcid.org/0000-0002-3878-0861; slavaromash@gmail.com; Kharkov State Academy of Physical Culture; Klochkovskaya str. 99, Kharkov, 61022, Ukraine.

Podavalenko O.V.; http://orcid.org/0000-0001-9143-3351; alekontp@ukr.net; Kharkov State Academy of Physical Culture; Klochkovskaya str. 99, Kharkov, 61022, Ukraine.

Volodchenko O.A.; http://orcid.org/0000-0002-1189-3524; kh_alex.kick@ukr.net; Kharkov State Academy of Physical Culture; Klochkovskaya str. 99, Kharkov, 61022, Ukraine.

Volodchenko J.A.; http://orcid.org/ 0000-0001-5359-4533; julia_simi@ukr.net; Kharkov State Academy of Physical Culture; Klochkovskaya str. 99, Kharkov, 61022, Ukraine.

\section{Cite this article as:}

Podrigalo OO, Borisova OV, Podrigalo LV, lermakov SS, Romanenko VV, Podavalenko OV, Volodchenko OA, Volodchenko JO. Comparative analysis of the athletes' functional condition in cyclic and situational sports. Physical education of students, 2019;23(6):313-319.

https://doi.org/10.15561/20755279.2019.0606

This is an Open Access article distributed under the terms of the Creative Commons Attribution License, which permits unrestricted use, distribution, and reproduction in any medium, provided the original work is properly cited http://creativecommons.org/licenses/by/4.0/deed.en

Received: 26.10.2019

Accepted: 30.11.2019; Published: 18.12.2019 